\title{
Anion conducting chitosan/poly[(3-methyl-1-vinylimidazolium methyl sulfate)-co-(1-vinylcaprolactam)-co-(1-vinylpyrrolidone)] membrane for alkaline anion-exchange membrane fuel cells
}

\author{
Bei Ao ${ }^{1}$, Yanan Wei ${ }^{1}$, Xiaofan $\mathrm{Hou}^{1}$, Keryn Lian $^{2^{*}}$ and Jinli Qiao ${ }^{1 *}$ \\ ${ }^{1}$ College of Environmental Science and Engineering, Donghua Universtiy, \\ 2999 Ren'min North Road, Shanghai 201620, China) \\ ${ }^{2}$ Department of Materials Science and Engineering., University of Toronto, \\ Toronto, Ontario, Canada
}

Corresponding author. qiaojl@dhu.edu.cn, keryn.lian@utoronto.ca

Tel: +86-21-67792379. Fax: +86-21-67792159

Key words: Anion-exchange membrane, fuel cell, cross-linking, ionic conductivity, water uptake, oxidation stability.

Abstract. Anion-exchange membrane composed of chitosan (CS) and poly[(3-methyl-1-vinylimidazolium methyl sulfate)-co-(1-vinylcaprolactam)-co-(1vinylpyrrolidone)] (PMVMS-co-VC-co-VP) was prepared by applying double cross-linking technique for alkaline membrane fuel cell (AMFC). Having tested the ion conductivity and water uptake without thermal cross-linking, the CS/PMVMS-co-VC-co-VP-OH membrane (1:0.75 by mass) shows the ionic conductivity of $1.39 \times 10^{-3} \mathrm{~S} \mathrm{~cm}^{-1}$ with high water uptake of $88.45 \%$. However, when the membrane was double cross-linked by both thermal and chemical cross-linking, the ionic conductivity of the membrane was improved and reached to $1.58 \times 10^{-3} \mathrm{~S} \mathrm{~cm}^{-1}$, instead, the water uptake of the membrane was largely decreased to lower than $48.02 \%$. The oxidation stability of the membrane was also enhanced greatly. Moreover, the ionic conductivity increases as the temperature increased; with the maximum $\mathrm{OH}^{-}$conductivity of $3.85 \times 10^{-3} \mathrm{~S} \mathrm{~cm}^{-1}$ was obtained at $80^{\circ} \mathrm{C}$.

\section{Introduction}

Fuel cell technologies are at the forefront of the green energy, especially for alkaline anion-exchange membrane (AEM) fuel cells which has been investigated for a few years [1]. When the operational environment is alkaline, AMFCs have advantages of faster electrokinetics, lower fuel crossover, reduced $\mathrm{CO}$ poisoning, and use of non-precious metal catalysts such as silver, nickel and perovskite [2]. To date, much efforts have been taken to develop various types of AEMs based on quaternized polymers such as polysiloxane [3] and radiation grafted [VPSIm] [OH] [4]. In this article, a new type of alkaline anion-exchange membrane was proposed based on cross-linking chitosan (CS) and poly[(3-methyl-1-vinylimidazolium methyl sulfate)-co-(1-vinylcaprolactam)-co-(1-vinylpyrrolidone)] composite (CS/PMVMS-co-VC-co-VP). The anion conducting membranes based on chitosan have garnered lots of interest for its excellent film-forming characteristic, controllable water uptake, and good mechanical property [5]. On the other hand, PMVMS-co-VC-co-VP, a water-soluble quaternized copolymer, could offer anions as charge carriers to conduct hydroxide. Considering that CS and PMVMS-co-VC-co-VP are both highly watersoluble, a major research challenge should be using the cross-linking technique to fix 
the CS and PMVMS-co-VC-co-VP in stable structures while maintain its high $\mathrm{OH}^{-}$conductivity and low water uptake. In this work, the technique of thermal and chemical cross-linking is applied to modify the CS and PMVMS-co-VC-co-VP blended polymer membrane and the properties for fuel cell potential application are studied.

\section{Experimental}

Materials and membrane preparation. The membrane was prepared by a simple solution-casting method as we have reported elsewhere [6,7]. $2 \mathrm{~g}$ chitosan (CS) was first dissolved into 100ml of 5\% aqueous solution of acetic acid. Then, appropriate amounts of PMVMS-co-VC-co-VP (20\% water solution, Aldrich) and above CS were mixed in the selected blend ratios (1:0.25, 1:0.5, 1:0.75 and 1:1 in mass). The resulting solution was poured into plastic petri dishes, and water was evaporated under ambient conditions. Cross-linking process was proceeded by the two following ways: (i) the membranes were first thermal treated at $140{ }^{\circ} \mathrm{C}$ for an hour to induce physical cross-linking between $\mathrm{CS}$ chains. Then the membranes were immersed into the cross-linking reaction solution consisting of $10 \mathrm{wt} \%$ glutaraldehyde (GA) for further chemical cross-linking for another $1 \mathrm{~h}$. (ii) The membranes were immersed into the cross-linking reaction solution for direct chemical cross-linking for $1 \mathrm{~h}$. Before the use, the membranes were treated by immersing in $2.0 \mathrm{M} \mathrm{KOH}$ solution for $24 \mathrm{~h}$, then rinsed repeatedly with deionized (D.I.) water to remove the absorbed $\mathrm{KOH}$ on membrane surfaces for final ionic conductivity measurements. Fig. 1 shows a model of the inner structure of CS /PMVMS-co-VC-co-VP-OH ${ }^{-}$membrane. It can be seen that the interpenetrating network structure forming between the CS chains makes the membrane more stable.

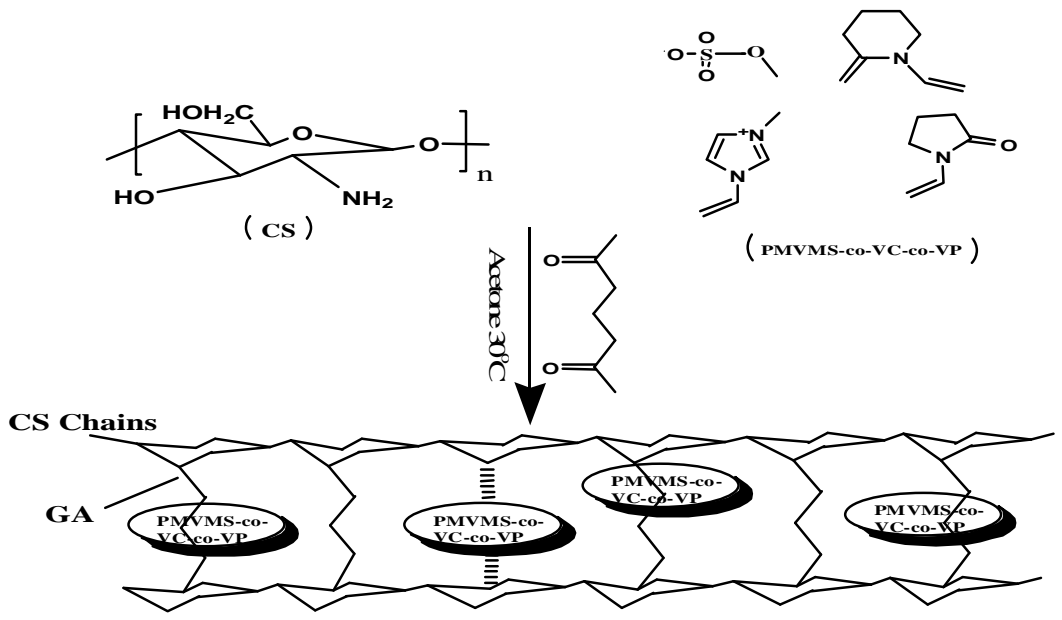

Fig. 1 Schematic structures of Chitosan, PMVMS-co-VC-co-VP and cross-linking of CS/ PMVMS-co-VC-co-VP-OHª alkaline membranes.

Ionic conductivity and water uptake. Before testing, the membrane sample was cut to be the size of $1.3 \times 0.8 \mathrm{~cm}$, and assured the membrane in the environment of D. I. water. The PH of the membrane surface was natural. The impedance was measured by placing the cell in a temperature range of $30 \sim 80^{\circ} \mathrm{C}$. The ionic conductivity was calculated by the following expression:

$$
\sigma=\frac{l}{R T W}
$$

where $l$ is the length of the membrane between two potential sensing platinum wires, $R$ is the 
membrane resistance, $W$ and $T$ are the width and the thickness of the membrane, respectively.

The swelling of the membranes was evaluated by the water uptake (WU) of the membranes, which was estimated from the mass change before and after the complete dryness of the membrane. A dry membrane was swelled in D.I. water for a day, then the surface water was wiped carefully with a filter paper, and it was immediately weighed. After drying the membrane overnight in a vacuum oven at $60^{\circ} \mathrm{C}$, the WU value was calculated using the expression:

$$
\mathrm{WU}=\frac{W_{w e t}-W_{d r y}}{W_{d r y}}
$$

Where $\mathrm{W}_{\text {wet }}$ and $\mathrm{W}_{\mathrm{dry}}$ are the masses of fully hydrated membrane and the dry membrane, respectively.

\section{Results and Discussion}

Ionic conductivity and water uptake property. In order to investigate the best suitable composition for membrane preparation and the thermal cross-linking effect on the CS/PMVMS-co-VC-co-VP membrane performance, it was assessed by Fig. $2(\mathrm{a})$, which shows the $\mathrm{OH}^{-}$conductivity $\left(\sigma_{\mathrm{OH}}{ }^{-}\right)$and water uptake (WU) of CS /PMVMS-co-VC-co-VP-OH- membrane with only chemical cross-linking modification and Fig. 2 (b) with both thermal and chemical cross-linking modifications. It can be seen that the maximum $\sigma_{\mathrm{OH}}{ }^{-}$value measured at $25{ }^{\circ} \mathrm{C}$ with $\mathrm{AC}$ impedance spectroscopy reaches as high as $1.49 \times 10^{-3} \mathrm{~S} \mathrm{~cm}^{-1}$ at a CS/PMVMS-co-VC-co-VP polymer composition of 1:0.75 by mass with only chemical cross-linking modification and then decreased. This can be attributed to the increased number of charge carriers from the PMVMS-co-VC-co-VP domains. In fact, CS can be thermally cross-linking by heat treatment, thus the density of membranes can be increased by annealing under high temperature for a certain period of time. Annealing can also help to increase the thermal stability of the membrane. To clarify this point, In Fig. 2(b), the double cross-linking modification was further conducted. Similar to the results of Fig. 2(a), the $\mathrm{OH}^{-}$conductivity of the membrane increased as the mass ratio of CS/PMVMS-co-VC-co-VP increases. However, the water uptake of the membrane significantly decreased from $88.45 \%$ reached $48.02 \%$, while the $\mathrm{OH}^{-}$ conductivity increased to $1.58 \times 10^{-3} \mathrm{~S} \mathrm{~cm}^{-1}$. Obviously, to obtain both good mechanical property and high conductivity of the membranes, in this work, both thermal cross-linking and chemical cross-linking are needed. 

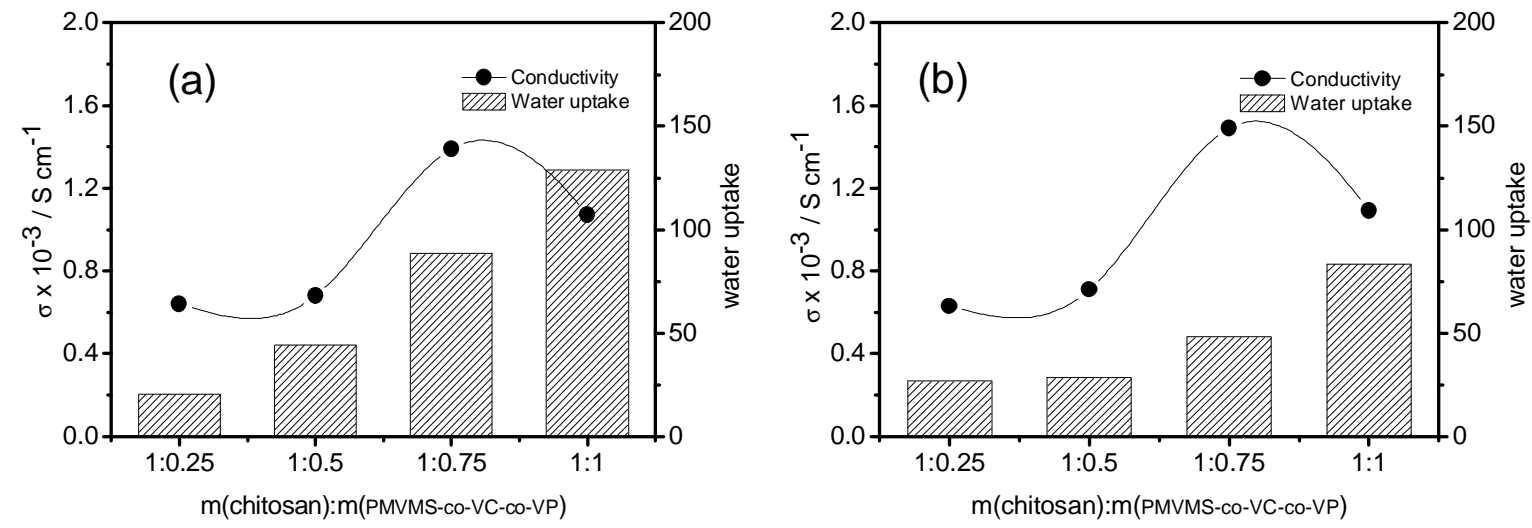

Fig. $2 \mathrm{OH}^{-}$conductivity and $W U$ of CS/PMVMS-co-VC-co-VP membranes as a function of CS and PMVMS-co-VC-co-VP mass ratio (a) with only chemical cross-linking and (b) with both thermal and chemical cross-linking under ambient conditions.

Oxidative stability. To further explore whether the thermal cross-linking influences the CS /PMVMS-co-VC-co-VP-OH ${ }^{-}$membrane, the oxidative stability of membrane at a mass ratio of 1:0.75 was tested with double cross-linking in contrast to the one without thermal cross-linking. It was noticed that the oxidative stability of the membrane was largely improved after the double cross-linking modifications. After 96 hours, the mass of the membrane sample after double cross-linking modification still remains nearly 2 times more than the one without thermal cross-linking. Apparently, by introducing the thermal cross-linking procedure can not only improve membrane conductivity and water uptake property but also prominently improve the CS /PMVMS-co-VC-co-VP-OH- membrane's oxidation stability.

Temperature dependences of $\mathrm{OH}^{-}$conductivity. Fig. 3(a) shows the correlation between conductivity and temperature for CS/PMVMS-co-VC-co-VP membrane (1:0.75 mass ratio) with both thermal and chemical cross-linking modifications. It can be seen that the $\mathrm{OH}^{-}$conductivity of the membrane was gradually increased with increasing temperature and the highest $\mathrm{OH}^{-}$conductivity of $3.85 \times 10^{-3} \mathrm{~S} \mathrm{~cm}^{-1}$ at $80^{\circ} \mathrm{C}$ was obtained. This is desirable when it is used as alkaline anion-exchange membrane in a fuel cell. Fig. 3(b) further shows the Arrhenius plots in temperature range from 30 to $80{ }^{\circ} \mathrm{C}$, where the $\ln \sigma$ is in line with 1000/T plot in all tested temperature range. According to the equation (3), where $\sigma_{0}$ is a pre-exponential factor, $E a$ is the apparent activation energy, and $T$ is the thermodynamic temperature in $K$.

$$
\sigma=\sigma_{0} \exp \left(-\frac{E a}{R T}\right)
$$

The $E_{a}$ can be estimated from the linear regression of $\log (\sigma)$ vs. $1000 /$ T. The values of $E_{a}$ thus obtained from the Arrhenius plot was $7.83 \mathrm{~kJ} \mathrm{~mol}^{-1}$, indicating that the Grotthus mechanism is mainly the conduction mechanism for CS /PMVMS-co-VC-co-VP-OH ${ }^{-}$membrane [8]. 

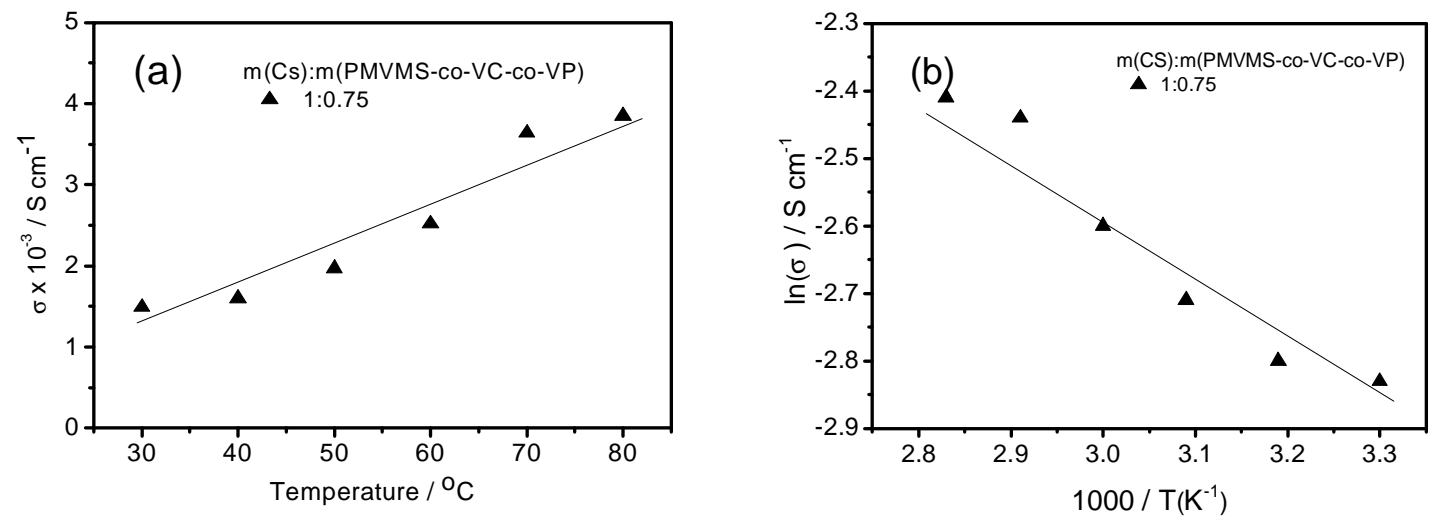

Fig. 3 (a) Temperature dependences of $\mathrm{OH}^{-}$conductivity; (b) the ln $\sigma$ vs. 1000/T plot for CS/PMVMS-co-VC-co-VP alkaline membrane. Polymer composition $\mathrm{CS} / \mathrm{PMVMS}-\mathrm{co}-\mathrm{VC}-\mathrm{co}-\mathrm{VP}=1: 0.75$ by mass.

\section{Conclusions}

In a word, the thermal cross-linking procedure was found to play a critical role in cutting down water uptake and improving the oxidation stability of the CS/PMVMS-co-VC-co-VP-OH membrane. By applying the double cross-linking techniques, the maximum $\mathrm{OH}^{-}$conductivity of $3.85 \times 10^{-3} \mathrm{~S} \mathrm{~cm}^{-1}$ was obtained at $80^{\circ}$, which is promising for potential use in fuel cells.

\section{Acknowledgements}

The authors thank for the financial support from the Innovation Program of Shanghai Municipal Education Commission (14ZZ074), the Project of Introducing Overseas Intelligence High Education of China (2016-2017) and the College of Environmental Science and Engineering, State Environmental Protection Engineering Center for Pollution Treatment and Control in Textile Industry, Donghua University.

\section{References}

[1] Y.J. Wang, J.L. Qiao, R. Baker and J.J. Zhang: Chem. Soc. Rev. Vol. 42 (2013), p. 5768-5787

[2] J. Zhou, M. Ünlü, I. Anestis-Richard and P.A. Kohl: J. Membr. Sci. Vol. 350 (2010), p. 286-292

[3] J.J. Kang, W.Y. Li and Y. Lin: Polymers for Advanced Technologies, Vol. 15 (2004), p. 61-64

[4] T. Feng, B. Lin, S. Zhang, N. Yuan, F. Chu, M. A. Hickner, C. Wang, L. Zhu and J. Ding: J. Membr. Sci. Vol. 508 (2016), p. 7-13

[5] J. Ma, N.A. Choudhury, Y. Sahai, A high performance direct borohydride fuel cell employing cross-linked chitosan membrane, Journal of Power Sources, 196(2011)8257-8264.

[6] J. Zhang, J.L. Qiao, L.L, Liu, Jiang G, Cross-linked poly(vinyl alcohol)/poly (diallyldimethylammonium chloride) as anion-exchange membrane for fuel cell applications, J. Power Sources. 240(2013)359-367.

[7] F.F. Song, Y.S. Fu, Y. Gao, J.D. Li, J.L. Qiao and X.-D. Zhou: Electrochim. Acta Vol. 177 (2015), p. 137-144

[8] J.L. Qiao, N. Yoshimoto and M. Morita: J. Power Sources Vol. 105 (2012), p. 45-51 